Few-Body Systems 0, 1-6 (2020)

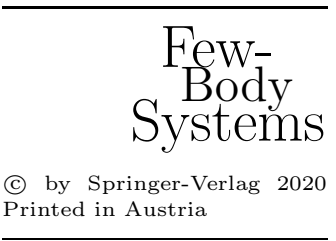

\title{
Recent Progress in Effective Field Theory in the One-Nucleon Sector
}

\author{
S. Scherer* \\ Institut für Kernphysik, Johannes Gutenberg-Universität Mainz, J. J. Becher-Weg 45, \\ D-55099 Mainz, Germany
}

\begin{abstract}
Chiral perturbation theory (ChPT) is the effective field theory of the strong interactions at low energies. We will address the issue of a consistent power counting scheme in a manifestly Lorentz-invariant formulation of baryon ChPT. As applications we show how the inclusion of vector and axial-vector mesons in the calculation of the nucleon electromagnetic and axial form factors, respectively, lead to an improved description of the empirical data. Finally, we will outline a systematic implementation of the $\Delta(1232)$ resonance into the effective field theory program.
\end{abstract}

\section{Introduction}

Effective field theory (EFT) is a powerful tool for describing the strong interactions at low energies. The EFT of the interactions among the Goldstone bosons of spontaneous chiral symmetry breaking in QCD is (mesonic) chiral perturbation theory (ChPT) [1, 2] (see, e.g., Ref. [3] for a pedagogical introduction). Besides the most general Lagrangian a successful EFT program requires a consistent power counting scheme to assess the importance of a given renormalized diagram. In the following we will outline some recent developments in devising a renormalization scheme leading to a simple and consistent power counting for manifestly Lorentz-invariant baryon ChPT [4]. The approach allows for both the inclusion of further degrees of freedom beyond pions and nucleons [5, 6] and the application to higher-loop calculations [7, 8].

\section{Renormalization and Power Counting}

The Lagrangian of the most general chirally invariant interaction of pions and nucleons in the one-nucleon sector is organized in a derivative and quark-mass expansion [1, 2, 3, 4]:

$$
\mathcal{L}_{\text {eff }}=\mathcal{L}_{\pi}+\mathcal{L}_{\pi N}=\mathcal{L}_{\pi}^{(2)}+\mathcal{L}_{\pi}^{(4)}+\cdots+\mathcal{L}_{\pi N}^{(1)}+\mathcal{L}_{\pi N}^{(2)}+\cdots
$$

\footnotetext{
*E-mail address: scherer@kph.uni-mainz.de
} 
The aim is to devise a renormalization procedure generating, after renormalization, the following power counting: a loop integration in $n$ dimensions counts as $q^{n}$, pion and fermion propagators count as $q^{-2}$ and $q^{-1}$, respectively, vertices derived from $\mathcal{L}_{\pi}^{2 k}$ and $\mathcal{L}_{\pi N}^{(k)}$ count as $q^{2 k}$ and $q^{k}$, respectively. Here, $q$ generically denotes a small expansion parameter such as, e.g., the pion mass.

In order to illustrate the issue of power counting, we consider as an example the one-loop integral (in the chiral limit)

$$
H\left(p^{2}, m^{2} ; n\right)=\int \frac{d^{n} k}{(2 \pi)^{n}} \frac{i}{\left[(k-p)^{2}-m^{2}+i 0^{+}\right]\left[k^{2}+i 0^{+}\right]},
$$

where $\Delta=\left(p^{2}-m^{2}\right) / m^{2}=\mathcal{O}(q)$ is a small quantity. Applying the dimensional counting analysis of Ref. [9], the result of the integration is of the form

$$
H \sim F(n, \Delta)+\Delta^{n-3} G(n, \Delta)
$$

where $F$ and $G$ are hypergeometric functions which can be expanded in $\Delta$ for any $n$. In the present case, we want the renormalized integral to be of the order $D=n-1-2=n-3$.

The infrared regularization of Becher and Leutwyler [10] makes use of the Feynman parametrization

$$
\frac{1}{a b}=\int_{0}^{1} \frac{d z}{[a z+b(1-z)]^{2}}
$$

with $a=(k-p)^{2}-m^{2}+i 0^{+}$and $b=k^{2}+i 0^{+}$. The resulting integral over the Feynman parameter $z$ is then rewritten as

$$
H=\int_{0}^{1} d z \cdots=\int_{0}^{\infty} d z \cdots-\int_{1}^{\infty} d z \cdots
$$

where the first, so-called infrared (singular) integral satisfies the power counting, while the remainder violates power counting but turns out to be regular and can thus be absorbed in counterterms.

The central idea of the extended on-mass-shell (EOMS) scheme [11 consists of performing additional subtractions beyond the MS scheme of Ref. [4. Since the terms violating the power counting are analytic in small quantities, they can be absorbed by counterterm contributions. To that end one first expands the integrand in small quantities and subtracts those (integrated) terms whose order is smaller than suggested by the power counting. The corresponding subtraction term reads

$$
H^{\mathrm{subtr}}=\left.\int \frac{d^{n} k}{(2 \pi)^{n}} \frac{i}{\left[k^{2}-2 p \cdot k+i 0^{+}\right]\left[k^{2}+i 0^{+}\right]}\right|_{p^{2}=m^{2}}
$$

and the renormalized integral is written as $H^{R}=H-H^{\text {subtr }}=\mathcal{O}(q)$ as $n \rightarrow 4$.

In Ref. [12] the IR regularization of Becher and Leutwyler was reformulated in a form analogous to the EOMS renormalization scheme. Within this (new) 
formulation the subtraction terms are found by expanding the integrands of loop integrals in powers of small parameters (small masses and Lorentz-invariant combinations of external momenta and large masses) and subsequently exchanging the order of integration and summation. The new formulation of IR regularization can be applied to diagrams with an arbitrary number of propagators with various masses (e.g., resonances) and/or diagrams with several fermion lines as well as to multi-loop diagrams.

\section{Applications}

\subsection{Electromagnetic Form Factors}

The nucleon matrix element of the electromagnetic current operator $J^{\mu}(x)$ can be parameterized in terms of two form factors,

$$
\left\langle N\left(p^{\prime}\right)\left|J^{\mu}(0)\right| N(p)\right\rangle=\bar{u}\left(p^{\prime}\right)\left[F_{1}^{N}\left(Q^{2}\right) \gamma^{\mu}+i \frac{\sigma^{\mu \nu} q_{\nu}}{2 m_{p}} F_{2}^{N}\left(Q^{2}\right)\right] u(p), \quad N=p, n,
$$

where $q=p^{\prime}-p, Q^{2}=-q^{2}$, and $m_{p}$ is the proton mass. At $Q^{2}=0$, the so-called Dirac and Pauli form factors $F_{1}$ and $F_{2}$ reduce to the charge and anomalous magnetic moment in units of the elementary charge and the nuclear magneton $e /\left(2 m_{p}\right)$, respectively: $F_{1}^{p}(0)=1, F_{1}^{n}(0)=0, F_{2}^{p}(0)=1.793$, and $F_{2}^{n}(0)=$ -1.913 . The Sachs form factors $G_{E}$ and $G_{M}$ are linear combinations of $F_{1}$ and $F_{2}$,

$G_{E}^{N}\left(Q^{2}\right)=F_{1}^{N}\left(Q^{2}\right)-\frac{Q^{2}}{4 m_{p}^{2}} F_{2}^{N}\left(Q^{2}\right), \quad G_{M}^{N}\left(Q^{2}\right)=F_{1}^{N}\left(Q^{2}\right)+F_{2}^{N}\left(Q^{2}\right), \quad N=p, n$.

It has been known for some time that ChPT results at $\mathcal{O}\left(q^{4}\right)$ only provide a decent description up to $Q^{2}=0.1 \mathrm{GeV}^{2}$ and do not generate sufficient curvature for larger values of $Q^{2}$ [13, 14]. To improve these results higher-order contributions have to be included. This can be achieved by performing a full calculation at $\mathcal{O}\left(q^{5}\right)$ which would also include the analysis of two-loop diagrams. Another possibility is to include additional degrees of freedom, through which some of the higher-order contributions are re-summed. Both the reformulated IR regularization and the EOMS scheme allow for a consistent inclusion of vector mesons which already a long time ago were established to play an important role in the description of the nucleon form factors. Figure 1 shows the results for the electric and magnetic Sachs form factors in the EOMS scheme (solid lines) and the infrared renormalization (dashed lines) [15]. A consistent inclusion of vector mesons clearly improves the quality of the description.

\subsection{Axial and Induced Pseudoscalar Form Factors}

Assuming isospin symmetry, the most general parametrization of the isovector axial-vector current evaluated between one-nucleon states is given by

$$
\left\langle N\left(p^{\prime}\right)\left|A^{\mu, a}(0)\right| N(p)\right\rangle=\bar{u}\left(p^{\prime}\right)\left[\gamma^{\mu} \gamma_{5} G_{A}\left(Q^{2}\right)+\frac{q^{\mu}}{2 m_{N}} \gamma_{5} G_{P}\left(Q^{2}\right)\right] \frac{\tau^{a}}{2} u(p),
$$



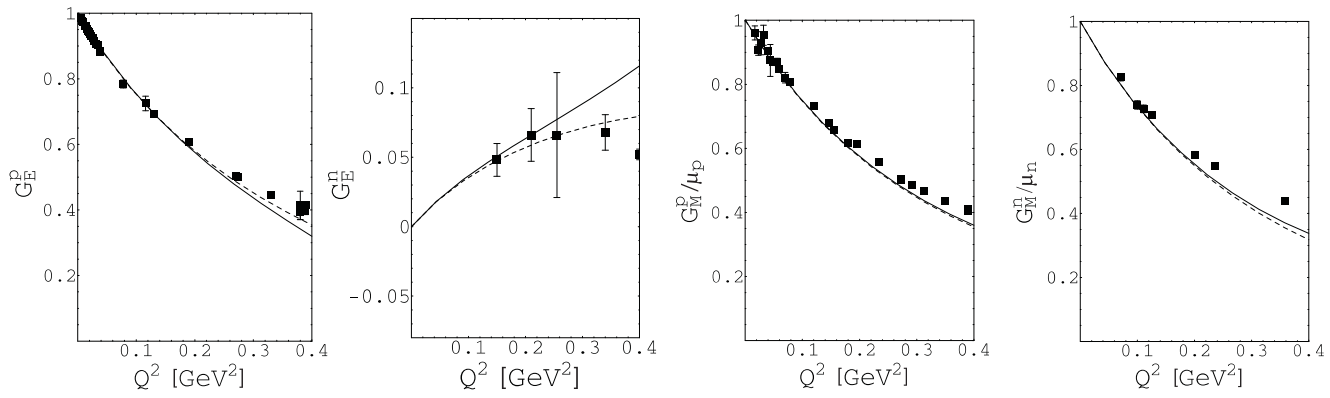

Figure 1. The Sachs form factors of the nucleon in manifestly Lorentz-invariant chiral perturbation theory at $\mathcal{O}\left(q^{4}\right)$ including vector mesons as explicit degrees of freedom. Full lines: results in the extended on-mass-shell scheme; dashed lines: results in infrared regularization.

where $q_{\mu}=p_{\mu}^{\prime}-p_{\mu}, Q^{2}=-q^{2}$, and $m_{N}$ denotes the nucleon mass. $G_{A}\left(Q^{2}\right)$ is the axial form factor and $G_{P}\left(Q^{2}\right)$ is the induced pseudoscalar form factor. The value of the axial form factor at zero momentum transfer is defined as the axial-vector coupling constant, $g_{A}=G_{A}\left(Q^{2}=0\right)=1.2695(29)$, and is quite precisely determined from neutron beta decay. In Ref. [16] the form factors $G_{A}$ and $G_{P}$ have been calculated in manifestly Lorentz-invariant baryon chiral perturbation theory up to and including order $\mathcal{O}\left(q^{4}\right)$. In addition to the standard treatment including the nucleon and pions, the axial-vector meson $a_{1}$ has also been considered as an explicit degree of freedom. The inclusion of the axialvector meson effectively results in one additional low-energy coupling constant which has been determined by a fit to the data for $G_{A}\left(Q^{2}\right)$. The inclusion of the axial-vector meson results in an improved description of the experimental data for $G_{A}$ (see Fig. 2).

\subsection{The Delta Resonance}

A relativistic description of the spin-3/2 delta resonance typically starts with the Rarita-Schwinger approach [17] in terms of a vector-spinor field $\Psi^{\mu}$. Such a description involves too many dynamical degrees of freedom resulting in constraints. In the EFT program one needs to construct the most general interaction Lagrangian. When dealing with systems involving constraints one has to make sure that the corresponding Lagrangian equations of motion do not involve an inconsistency. This is achieved with the aid of Dirac's analysis [18 using the Hamiltonian method. In a system with constraints there exist certain relations connecting the momentum variables, of the type $\Phi_{m}(q, p)=0$ which are referred to as primary constraints. Introducing the constraints in terms of Lagrange multipliers into the Hamiltonian, $H_{T}=H+u_{m} \Phi_{m}$, one considers the time evolution in terms of Poisson brackets, $\left\{H_{T}, \Phi_{m}\right\}=0$, thus generating new (secondary) constraints. The procedure is iterated until all Lagrange multipliers have been solved. In a consistent theory the number of initial degrees of freedom minus the number of constraints must equal the correct number of degrees of freedom. From this one obtains restrictions on the possible interaction terms. 


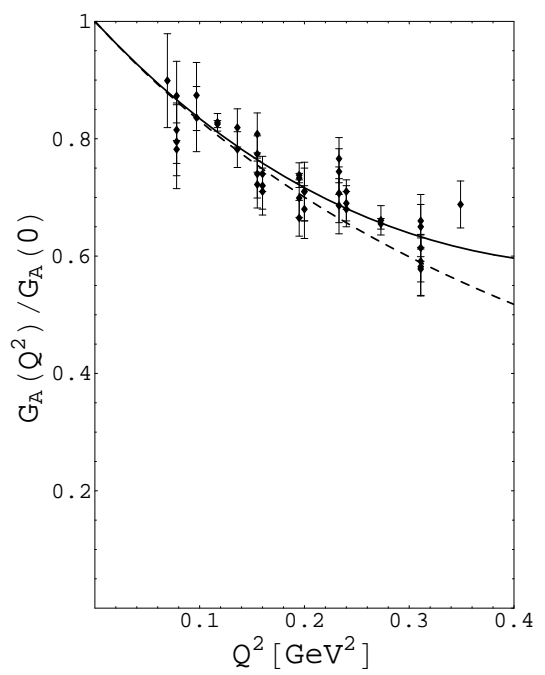

Figure 2. Axial form factor $G_{A}$ at $\mathcal{O}\left(q^{4}\right)$ including the axial-vector meson $a_{1}$ explicitly. Full line: result in infrared renormalization, dashed line: dipole parametrization.

For example, the $\mathcal{L}_{\pi \Delta}$ interaction Lagrangian [19]

$$
\mathcal{L}_{\pi \Delta}=-\bar{\Psi}^{\mu}\left[\frac{g_{1}}{2} g_{\mu \nu} \gamma^{\alpha} \gamma_{5} \partial_{\alpha} \phi+\frac{g_{2}}{2}\left(\gamma_{\mu} \partial_{\nu} \phi+\partial_{\mu} \phi \gamma_{\nu}\right) \gamma_{5}+\frac{g_{3}}{2} \gamma_{\mu} \gamma^{\alpha} \gamma_{5} \gamma_{\nu} \partial_{\alpha} \phi\right] \Psi^{\nu}
$$

contains three seemingly independent coupling constants. However, an analysis of the constraints yields [20]

$$
g_{2}=A g_{1}, \quad g_{3}=-\frac{1}{2}\left(1+2 A+3 A^{2}\right) g_{1},
$$

where $A$ is a parameter of the lowest-order Lagrangian. As a result of these constraints the total Lagrangian is invariant under the so-called point transformation, guaranteeing that the physical quantities are independent of the offshell parameter $A$. On the other hand, demanding the invariance under the point transformation alone is less stringent and produces only a class of relations among the coupling constants. The analysis of the constraint as a rule leads to a reduction in the number of free parameters of the Lagrangian.

\section{Summary and Conclusions}

Both the infrared regularization and the EOMS scheme allow for a simple and consistent power counting in manifestly Lorentz-invariant baryon chiral perturbation theory. The inclusion of vector and axial-vector mesons as explicit degrees of freedom leads to an improved phenomenological description of the electromagnetic and axial form factors, respectively. When dealing with the delta resonance, Dirac's constraint analysis leads to an identification of redundant parameters. 
Acknowledgement. I would like to thank D. Djukanovic, T. Fuchs, J. Gegelia, C. Hacker, G. Japaridze, M. R. Schindler, and N. Wies for the fruitful collaboration on the topics of this talk. This work was made possible by the financial support from the Deutsche Forschungsgemeinschaft (SFB 443 and SCHE 459/2-1) and the EU Integrated Infrastructure Initiative Hadron Physics Project (contract number RII3-CT-2004-506078).

\section{References}

1. Weinberg, S.: Physica A96, 327 (1979)

2. Gasser, J., Leutwyler, H.: Annals Phys. 158, 142 (1984)

3. Scherer, S.: Adv. Nucl. Phys. 27, 277 (2003); Scherer, S., Schindler, M. R.: arXiv:hep-ph/0505265

4. Gasser, J., Sainio, M. E., Švarc, A.: Nucl. Phys. B307, 779 (1988)

5. Fuchs, T., Schindler, M. R., Gegelia, J., Scherer, S.: Phys. Lett. B575, 11 (2003)

6. Hacker, C., Wies, N., Gegelia, J., Scherer, S.: Phys. Rev. C72, 055203 (2005)

7. Schindler, M. R., Gegelia, J., Scherer, S.: Nucl. Phys. B682, 367 (2004)

8. Schindler, M. R., Djukanovic, D., Gegelia, J., Scherer, S.: Phys. Lett. B649, $390(2007)$

9. Gegelia, J., Japaridze, G. S., Turashvili, K. S.: Theor. Math. Phys. 101, 1313 (1994)

10. Becher, T., Leutwyler, H.: Eur. Phys. J. C9, 643 (1999)

11. Fuchs, T., Gegelia, J., Japaridze, G., Scherer, S.: Phys. Rev. D68, 056005 (2003)

12. Schindler, M. R., Gegelia, J., Scherer, S.: Phys. Lett. B586, 258 (2004)

13. Kubis, B., Meißner, U.-G.: Nucl. Phys. A679, 698 (2001)

14. Fuchs, T., Gegelia, J., Scherer, S.: J. Phys. G30, 1407 (2004)

15. Schindler, M. R., Gegelia, J., Scherer, S.: Eur. Phys. J. A26, 1 (2005)

16. Schindler, M. R., Fuchs, T., Gegelia, J., Scherer, S.: Phys. Rev. C75, 025202 (2007)

17. Rarita, W., Schwinger, J. S.: Phys. Rev. 60, 61 (1941)

18. Dirac, P. A. M.: Lectures on Quantum Mechanics. Mineola, NY: Dover 2001

19. Hemmert, T. R., Holstein, B. R., Kambor, J.: J. Phys. G24, 1831 (1998)

20. Wies, N., Gegelia, J., Scherer, S.: Phys. Rev. D73, 094012 (2006) 